\title{
The pressure tensor in tangential equilibria
}

\author{
F. Mottez \\ Centre d'études des Environnements Terrestre et Planétaires, CETP/IPSL/CNRS, Vélizy, France \\ Received: 20 February 2004 - Revised: 4 May 2004 - Accepted: 12 May 2004 - Published: 7 September 2004
}

\begin{abstract}
The tangential equilibria are characterized by a bulk plasma velocity and a magnetic field that are perpendicular to the gradient direction. Such equilibria can be spatially periodic (like waves), or they can separate two regions with asymptotic uniform conditions (like MHD tangential discontinuities). It is possible to compute the velocity moments of the particle distribution function. Even in very simple cases, the pressure tensor is not isotropic and not gyrotropic. The differences between a scalar pressure and the pressure tensor derived in the frame of the Maxwell-Vlasov theory are significant when the gradient scales are of the order of the Larmor radius; they concern mainly the ion pressure tensor.
\end{abstract}

Key words. Magnetospheric physics (magnetopause, cusp and boundary layers) - Space plasma physics (discontinuities; kinetic and MHD theory)

\section{Introduction}

We define tangential equilibria as monodimensional equilibrium structures, where the magnetic field is perpendicular to the gradient direction and the plasma velocity along the gradient direction is null. When the magnetic field does not keep a constant direction, these solutions are sometimes referred to as sheared equilibria. These equilibria can be described by MHD or multifluid theories (for example, as tangential discontinuities), but if the gradients are sharp in comparison to one ion Larmor gyroradius, the fluid models cannot look inside these structures.

Sharp tangential equilibria are often met in space collisionless plasmas. A solar wind tangential discontinuity was crossed by the Cluster spacecraft and its thickness was estimated to 600-1000 km (Dunlop et al., 2002). The magnetic field amplitude was $30 \mathrm{nT}$; estimating an ion temperature of $100 \mathrm{eV}$, the thickness of the tangential discontinuity was less than three ion Larmor radii. The magnetopause is sometimes found to be a tangential discontinuity, it generally has a similar thickness. Tangential discontinuities exist also inside the Earth's magnetosphere: tangential current sheet crossings by Cluster allowed an estimated thickness of $1126 \mathrm{~km}$, and even $400 \mathrm{~km}$ (Petrukovich et al., 2003), that is, of the order of the

Correspondence to: F. Mottez

(fabrice.mottez@cetp.ipsl.fr) ion Larmor radius. Plasma cavities in the auroral zone and in the solar wind have a tangential equilibria geometry, and the density gradient sometimes do not exceed $1.4 \mathrm{~km}$, that is, two or three ion Larmor radii (Hilgers et al., 1992).

Theoretical works on tangential equilibria have shown the existence of analytical isothermal (Harris, 1962; Channell, 1976), or non isothermal solutions (Attico and Pegoraro, 1999). Other works, mainly focused on the study of the Earth's magnetopause and reviewed by Roth et al. (1996), have shown solutions that satisfy a larger class of constraints, but where the differential equations are solved numerically. Another class of tangential equilibria (Mottez, 2003) can explain the non isothermal equilibrium of deep plasma cavities in the Earth's auroral zone. All these equilibria have been developed in the frame of the collisionless plasma kinetic theory; they are solutions of the Maxwell-Vlasov equations. They all suppose particle distribution functions given, for each species $s$ by

$f_{s}=\int_{a_{1}}^{a_{2}} d a\left(\frac{\alpha_{a s}}{\pi}\right)^{3 / 2} e^{\left(-\frac{E}{T_{a s}}\right)} G_{a s}\left(p_{y}, p_{z}\right)$,

where $a$ is a scalar referring to an isothermal (trapped or passing) particle population, $a_{1}$ and $a_{2}$ are arbitrary (sometimes infinite), and $\alpha_{a s}=\left(m_{s} / 2 T_{a s}\right)^{1 / 2}$. The choice $G_{a s}$ is specific to each solution (Mottez, 2003). The variables

$$
\begin{aligned}
p_{y}=v_{y}+\frac{q}{m} A_{y}(x) & =v_{y}+\frac{q}{m} A(x) \cos \theta(x) \\
p_{z}=v_{z}+\frac{q}{m} A_{z}(x) & =v_{y}+\frac{q}{m} A(x) \sin \theta(x) \\
E & =v_{x}^{2}+v_{y}^{2}+v_{z}^{2}+\frac{2 q}{m} \Phi(x)
\end{aligned}
$$

are the invariants of the particle motion in the monodimensional equilibrium $\left(\partial_{y}=\partial_{z}=0, \partial_{t}=0\right)$.

Many studies have been devoted to the stability of these equilibria. They are generally based on MHD, Hall-MHD, or multifluid equations. What do we lose when we jump from the kinetic Vlasov theory to the fluid approach? We shall investigate this question through an evaluation of the fluid moments of the tangential equilibria; we will concentrate especially on the pressure tensor. 


\section{The basic equations of tangential equilibria}

We consider monodimensional equilibria, $y$ and $z$ are the directions of invariance. In tangential equilibria $B_{x}=0$ but $B_{y}(x)$ and $B_{z}(x)$ are functions of $x$. As $\partial_{t}=0$, the electric field derives from a scalar potential $\Phi(x)$, and only $E_{x}(x)$ can be different from zero. The vector potential has two components $A_{y}(x), A_{z}(x)$, and

$$
\begin{aligned}
& B_{z}(x)=d_{x} A_{y} \\
& B_{y}(x)=-d_{x} A_{z} .
\end{aligned}
$$

Since the particle distribution functions given in Eq. (1) depend only on the invariants of the motion of individual particles, they are solutions of the Vlasov equation. If $T_{a s}$ is independent of $a$, the equilibrium is isothermal and $T_{a s}$ is the temperature of the species $s$. We consider plasmas formed of an electron population $(s=e)$ and one ion species $(s=i)$. In order not to overload the equations, we express the dependence of the parameters on $s$ only when two different species are treated in the same equation.

The equilibria must also verify the Maxwell equations. For tangential equilibria, they are particularly simple. The Ampere condition is

$$
\begin{gathered}
J_{y}=-\frac{1}{\mu_{0}} d_{x} B_{z}=-\frac{1}{\mu_{0}} d_{x^{2}}^{2} A_{y} \\
J_{z}=\frac{1}{\mu_{0}} d_{x} B_{y}=-\frac{1}{\mu_{0}} d_{x^{2}}^{2} A_{z} .
\end{gathered}
$$

The contribution of each species to the current density $J_{y}$ is

$J_{y}(x)=q \int d \boldsymbol{v} v_{y} f$,

with a similar equation for $J_{z}$. The dependence of $J_{y}$ in $x$ comes from the dependence of $f$ on $E, p_{y}$ and $p_{z}$, which themselves depend on the scalar and vector potentials $\Phi(x), A_{y}(x)$ and $A_{z}(x)$. The Poisson equation writes

$\frac{d^{2} \Phi(x)}{d x^{2}}=-\frac{e}{\epsilon_{0}}\left(n_{i}(x)-n_{e}(x)\right)$,

where

$n_{i}=\int d \boldsymbol{v} f_{i}$ and $n_{e}=\int d \boldsymbol{v} f_{e}$.

\section{The first velocity moments of the distribution function}

We can express the total energy $E$ in Eq. (1) as the sum of the electric and kinetic energy:

$$
f=\int_{a_{1}}^{a_{2}} d a\left(\frac{\alpha_{a}}{\pi}\right)^{3 / 2} e^{\left(-\frac{2 \alpha_{a} q \Phi(x)}{m}\right)} e^{-\alpha_{a} v^{2}} G_{a}\left(p_{y}, p_{z}\right) .
$$

Eliminating $v_{y}$ and $v_{z}$ :

$$
\begin{aligned}
f= & \int_{a_{1}}^{a_{2}} d a\left(\frac{\alpha_{a}}{\pi}\right)^{3 / 2} e^{-\alpha_{a}\left[v_{x}^{2}+\left(p_{y}-\frac{q}{m} A_{y}\right)^{2}+\left(p_{z}-\frac{q}{m} A_{z}\right)^{2}\right]} \times \\
& G_{a}\left(p_{y}, p_{z}\right) e^{\left(-\frac{2 \alpha_{a} q \Phi}{m}\right)} .
\end{aligned}
$$

For a given particle species, the particle density is

$$
\begin{aligned}
n(x)= & \int_{a_{1}}^{a_{2}} d a\left(\frac{\alpha_{a}}{\pi}\right) \int e^{-\alpha_{a}\left[\left(p_{y}-\frac{q}{m} A_{y}\right)^{2}+\left(p_{z}-\frac{q}{m} A_{z}\right)^{2}\right]} \times \\
& G_{a}\left(p_{y}, p_{z}\right) e^{\left(-\frac{2 \alpha q \Phi(x)}{m}\right)} d p_{y} d p_{z},
\end{aligned}
$$

where $p_{y}$ and $p_{z}$ vary from $-\infty$ to $+\infty$. Let us define $n_{a}$ that depends on $x$ through the potentials $A_{y}(x), A_{z}(x)$, and $\Phi(x)$,

$$
\begin{aligned}
n_{a}(x)= & \left(\frac{\alpha_{a}}{\pi}\right) \int e^{-\alpha_{a}\left[\left(p_{y}-\frac{q}{m} A_{y}\right)^{2}+\left(p_{z}-\frac{q}{m} A_{z}\right)^{2}\right]} \times \\
& G_{a}\left(p_{y}, p_{z}\right) e^{\left(-\frac{2 \alpha_{a} q \Phi}{m}\right)} d p_{y} d p_{z} .
\end{aligned}
$$

Then,

$n(x)=\int_{a_{1}}^{a_{2}} d a n_{a}(x)$.

The contribution of a particle species to the current density $J_{y}$ is

$$
\begin{aligned}
J_{y}(x)= & q \int_{a_{1}}^{a_{2}} d a\left(\frac{\alpha_{a}}{\pi}\right) \int e^{\left(-\frac{2 \alpha_{a} q \Phi}{m}\right)}\left(p_{y}-\frac{q}{m} A_{y}\right) \times \\
& e^{-\alpha_{a}\left[\left(p_{y}-\frac{q}{m} A_{y}\right)^{2}+\left(p_{z}-\frac{q}{m} A_{z}\right)^{2}\right]} G_{a}\left(p_{y}, p_{z}\right) d p_{y} d p_{z} .
\end{aligned}
$$

The relation for $J_{z}$ is analogous. The integral $J_{x}$ includes the integral of the odd function $v_{x} \exp \left(-\alpha v_{x}^{2}\right)$; therefore, $J_{x}=0$.

Let us notice that

$$
\begin{aligned}
J_{y} & =\int_{a_{1}}^{a_{2}} d a q\left(\frac{m}{2 \alpha_{a} q}\right) \frac{\partial n_{a}}{\partial A_{y}}=\int_{a_{1}}^{a_{2}} d a T_{a} \frac{\partial n_{a}}{\partial A_{y}} \\
J_{z} & =\int_{a_{1}}^{a_{2}} d a T_{a} \frac{\partial n_{a}}{\partial A_{z}} .
\end{aligned}
$$

For a given species, the bulk velocity is simply its contribution to the current density divided by $q n$ :

$$
\begin{aligned}
& u_{y}=\frac{1}{\int_{a_{1}}^{a_{2}} d a n_{a}} \int_{a_{1}}^{a_{2}} d a \frac{T_{a}}{q} \frac{\partial n_{a}}{\partial A_{y}} \\
& u_{z}=\frac{1}{\int_{a_{1}}^{a_{2}} d a n_{a}} \int_{a_{1}}^{a_{2}} d a \frac{T_{a}}{q} \frac{\partial n_{a}}{\partial A_{z}} .
\end{aligned}
$$

The $u_{x}$ component, as $J_{x}$, is null.

\section{The pressure tensor}

By definition, the contribution of each species to the pressure tensor is

$\mathbf{p}=m \int(\boldsymbol{v}-\boldsymbol{u})(\boldsymbol{v}-\boldsymbol{u}) f d \boldsymbol{v}$,

where the tensor $(\boldsymbol{v}-\boldsymbol{u})(\boldsymbol{v}-\boldsymbol{u})$ is a dyadic product. Considering that the velocity $u_{x}$ is null,

$$
\begin{aligned}
p_{x x} & =m \int v_{x}^{2} f d v_{x} d p_{y} d p_{z} \\
& =m \iint_{a_{1}}^{a_{2}}\left\{\left(\frac{\alpha_{a}}{\pi}\right) \exp \left(-\frac{2 \alpha q \Phi}{m}\right) G_{a}\left(p_{y}, p_{z}\right) d p_{y} d p_{z}\right\} \times \\
& \left\{\left(\frac{\alpha_{a}}{\pi}\right)^{1 / 2} v_{x}^{2} e^{-\alpha_{a} v^{2}} d v_{x}\right\} d a .
\end{aligned}
$$


The terms between braces can be integrated separately. The first (integrated over $d p_{y} d p_{z}$ ) is $n_{a}$. The second (integrated over $\left.d v_{x}\right)$ is $\left(\frac{\alpha_{a}}{\pi}\right)^{1 / 2} \pi^{1 / 2} / 2 \alpha^{3 / 2}=\frac{T_{a}}{m}$. In the end,

$p_{x x}=\int_{a_{1}}^{a_{2}} d a n_{a} T_{a}$.

The computation of the other diagonal terms involves a finite bulk velocity,

$$
\begin{aligned}
p_{z z} & =\int_{a_{1}}^{a_{2}} d a m\left(\frac{\alpha_{a}}{\pi}\right) \int d p_{y} d p_{z} \times \\
& \left\{v_{z}^{2}-2 v_{z} u_{z}+u_{z}^{2}\right\} e^{-\alpha\left[v_{y}^{2}+v_{z}^{2}\right]} e^{\left(-\frac{2 \alpha q \Phi}{m}\right)} G_{a}\left(p_{y}, p_{z}\right) .
\end{aligned}
$$

The development of $\left(v_{z}-u_{z}\right)^{2}$ (in braces) can be cut into three parts, and $p_{z z}$ is the sum of the three corresponding integrals. The second and the third integrals are simply $-m n u_{z}^{2}$. The bulk velocity $u_{z}$ can be eliminated using Eq. (16). In the first integral, $v_{z}$ is eliminated with $p_{y}$ and $A_{y}$, and

$$
\begin{gathered}
\left(p_{z}-\frac{q}{m} A_{z}\right)^{2} e^{-\alpha_{a}\left[\left(p_{y}-\frac{q}{m} A_{y}\right)^{2}+\left(p_{z}-\frac{q}{m} A_{z}\right)^{2}\right]}= \\
{\left[\left(\frac{m}{2 \alpha_{a} q}\right)^{2} \frac{\partial^{2}}{\partial A_{z}^{2}}+\frac{1}{2 \alpha_{a}}\right] \cdot e^{-\alpha_{a}\left[\left(p_{y}-\frac{q}{m} A_{y}\right)^{2}+\left(p_{z}-\frac{q}{m} A_{z}\right)^{2}\right]} .}
\end{gathered}
$$

Therefore, Eq. (20) can also be written

$$
\begin{gathered}
p_{z z}=\int_{a_{1}}^{a_{2}} d a\left[T_{a} n_{a}+\frac{m T_{a}^{2}}{q^{2}} \frac{\partial^{2} n_{a}}{\partial A_{z}^{2}}\right] \\
-\frac{m}{\int d a n_{a}}\left[\int_{a_{1}}^{a_{2}} d a \frac{T_{a}}{q}\left(\frac{\partial n_{a}}{\partial A_{z}}\right)\right]^{2} .
\end{gathered}
$$

The relation for $p_{y y}$ is analogous (some minus signs appear at different places but the final result is the same). The offdiagonal terms $p_{x y}$ and $p_{x z}$ are equal to zero, because they include a product by the integral over $d v_{x}$ of an odd integrand. As

$$
\begin{gathered}
\left(p_{y}-\frac{q}{m} A_{y}\right)\left(p_{z}-\frac{q}{m} A_{z}\right) e^{-\alpha_{a}\left[\left(p_{y}-\frac{q}{m} A_{y}\right)^{2}+\left(p_{z}-\frac{q}{m} A_{z}\right)^{2}\right]} \\
=\left(\frac{m}{2 \alpha_{a} q}\right)^{2} \frac{\partial^{2}}{\partial A_{z} \partial A_{z}} \cdot e^{-\alpha_{a}\left[\left(p_{y}-\frac{q}{m} A_{y}\right)^{2}+\left(p_{z}-\frac{q}{m} A_{z}\right)^{2}\right]}
\end{gathered}
$$

the off-diagonal term $p_{y z}$, is

$$
\begin{aligned}
p_{y z}= & p_{z y}=\frac{m}{q^{2}}\left[\int_{a_{1}}^{a_{2}} d a T_{a}^{2} \frac{\partial^{2} n_{a}}{\partial A_{y} \partial A_{z}}\right. \\
& \left.\frac{1}{\int d a n_{a}^{2}} \int_{a_{1}}^{a_{2}} d a T_{a} \frac{\partial n_{a}}{\partial A_{y}} \int_{a_{1}}^{a_{2}} d a T_{a} \frac{\partial n_{a}}{\partial A_{z}}\right] .
\end{aligned}
$$

Obviously, the pressure tensor contains four different terms. For the sake of simplicity, let us consider the case of the isothermal equilibria, set when $T_{a}=T$ is constant. Then the pressure terms simplify into

$p_{x x}=n T$

$$
\begin{aligned}
& p_{y y}=n T\left[1+\frac{m T}{q^{2}} \frac{\partial}{\partial A_{y}}\left(\frac{1}{n} \frac{\partial n}{\partial A_{y}}\right)\right], \\
& p_{z z}=n T\left[1+\frac{m T}{q^{2}} \frac{\partial}{\partial A_{z}}\left(\frac{1}{n} \frac{\partial n}{\partial A_{z}}\right)\right], \\
& p_{y z}=n \frac{m T^{2}}{q^{2}} \frac{\partial}{\partial A_{y}}\left(\frac{1}{n} \frac{\partial n}{\partial A_{z}}\right)=n \frac{m T^{2}}{q^{2}} \frac{\partial}{\partial A_{z}}\left(\frac{1}{n} \frac{\partial n}{\partial A_{y}}\right) .
\end{aligned}
$$

If the magnetic field is everywhere parallel to $z$, the offdiagonal terms vanish, $p_{x x}$ and $p_{y y}$ represent the pressure components in the directions perpendicular to the magnetic field, and $p_{z z}=p_{/ /}$is the parallel component. The inequality $p_{z z} \neq p_{x x}$ shows that the pressure tensor is not isotropic. Moreover, the perpendicular terms are different $p_{x x} \neq p_{y y}$; therefore, the pressure tensor is non gyrotropic.

These non isotropic and non gyrotropic effects can be attributed to the finite Larmor radius $\rho_{L}$ : From a dimensional point of view, the terms $\left(p_{z z}-p_{x x}\right) / p_{x x}$ deduced from Eq. $(26-27)$, scale as $\left(\frac{m v_{\perp}}{q B}\right)^{2}=\left(\rho_{L} k\right)^{2}$, where $k$ is the inverse of the characteristic size $L$ of the density gradient. As long as $\frac{T_{i}}{T_{e}}<\frac{m_{i}}{m_{e}}$, the non isotropic and non gyrotropic terms are predominantely carried by the ions.

\section{First example: the Harris current sheet in the Earth's magnetotail}

In the simple example of the Harris current sheet, the pressure tensor can be expressed with elementary functions. This equilibrium corresponds to $G_{a}\left(p_{y}, p_{z}\right)=n_{0}+$ $\Delta_{0} n_{g} \exp v p_{y} / m$ in Eq. (9), where $\Delta_{0}$ is the Dirac distribution. The density $n_{0}$ is the density far from the discontinuity; it is arbitrary (it is null in the Harris paper (1962)). Defining $\delta=v\left(\frac{q}{m}\right)$, the magnetic field and the contribution of each particle species to the density are

$$
\begin{aligned}
B_{z}(x) & =-B_{0} \tanh \left(\frac{\delta B_{0} x}{2}\right) \\
n(x) & =n_{0}+N_{0} \exp \left(\delta A_{y}(x)\right)=n_{0}+\frac{N_{0}}{\cosh \left(\frac{\delta B_{0} x}{2}\right)^{2}} .
\end{aligned}
$$

The finite pressure components are $p_{x x}=p_{z z}=n T$ and

$$
p_{y y}=n T\left[1+m\left(\frac{\delta^{2} T}{q^{2}}\right) \frac{\left(n-n_{0}\right) n_{0}}{n^{2}}\right] \text {. }
$$

In the case $n_{0}=0$ of a bounded plasma (null density at infinity), the pressure tensor is a simple scalar tensor. It is a non gyrotropic tensor in all the other cases. Figure 1 shows an example of the Harris current sheet directly inspired from a tangential current sheet crossing studied by Petrukovich et al. (2003), and mentioned in the Introduction of this article. The parameters are inferred from the Cluster measurements: $T_{i}=1500 \mathrm{eV}, B_{0}=20 \mathrm{nT}, n_{0}=1.5 \mathrm{~cm}^{-3}$. We have set $\delta=1200 \mathrm{~m}^{-1} \mathrm{~T}^{-1}$, in order to set the layer thickness to $400 \mathrm{~km}$ (case of the 23:10 crossing (Petrukovich et al., 2003). We can see the magnetic field $B_{z}$ reversal on a scale of $400 \mathrm{~km}$. The the $p_{x x}=p_{z z}$ (dashed line) and $p_{y y}$ (continuous line) plasma pressure terms are significantly different, 

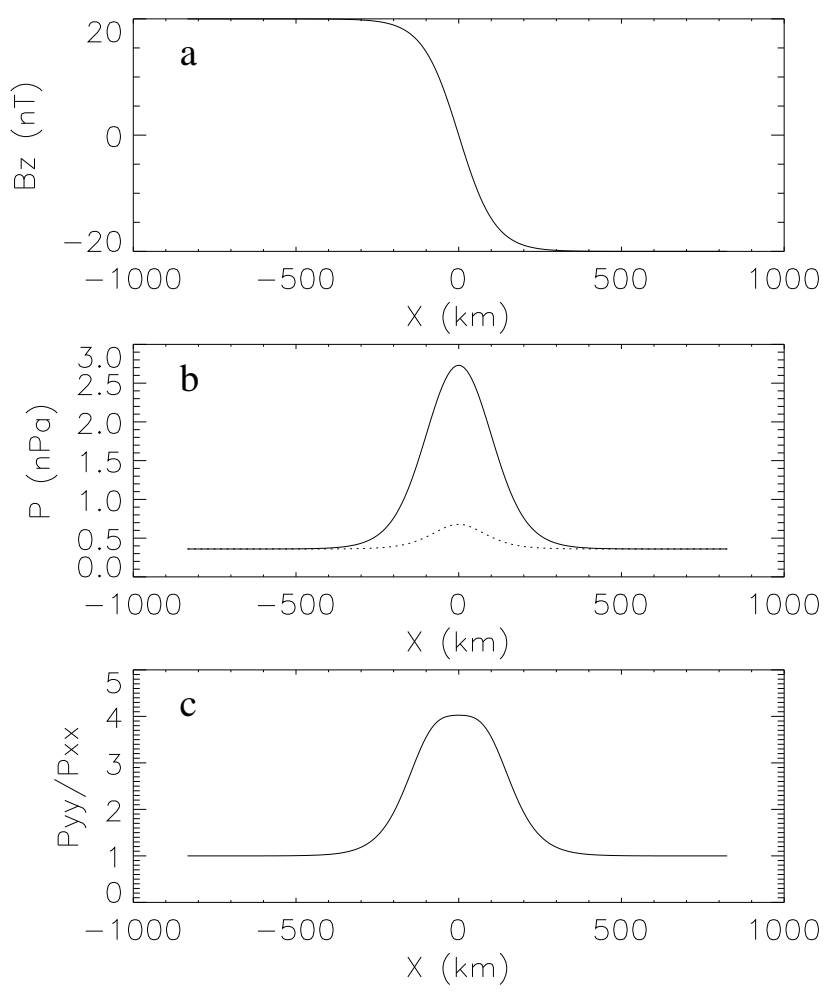

Fig. 1. The spatial dependence of physical parameters associated with a Harris current sheet: (a) the magnetic field $B_{z}$ in nano Tesla; (b) the pressure $p_{x x}=p_{z z}$ (dashed line) and $p_{y y}$ (continuous line) in nano Pascal; and (c) the ratio $p_{y y} / p_{x x}=p_{y y} / p_{z z}$. The $x$ component corresponds to the gradient direction, and $y$ and $z$ are tangential (it is not the GSM coordinate system). The parameters are inspired from a narrow current sheet crossing seen on board Cluster on 14 September 2001, at 23:10 UT, interpreted by Petrukovich et al. (2003) as a tangential discontinuity very similar to a Harris current sheet.

with their ratio reaches $p_{y y} / p_{x x}=p_{y y} / p_{z z}=4$ in the middle of the structure. The plasma is strongly non isotropic and non gyrotropic. In the case of a larger discontinuity, $1200 \mathrm{~km}$, (case of the 22:54 current sheet crossing), the ratio reaches a smaller value: 1.3. Although less impressive than in the previous case, the plasma pressure non gyrotropy cannot be neglected.

\section{Second example: density cavities in the Earth's auro- ral zone}

Let us now briefly examine the case of a density structure in a low $\beta$ plasma. Such structures where shown by Mottez (2003) to model the plasma cavities encountered in the high altitude Earth's auroral zone. A simple case corresponds to $G_{a}\left(p_{y}, p_{z}\right)=n_{c} \exp \left(-\eta\left(p_{y} / m\right)^{2}\right)$. In such structures, the size can reach the order of a few ion Larmor radii $\rho_{i}$, but cannot go below one ion Larmor radius. The magnetic field remains quasi-uniform, in spite of large plasma density vari- ations. With the (very accurate) approximation $A_{y}=B_{z} x$, the contribution of each particle species to the density is

$n(x)=n_{0}+N_{0} e^{-\xi B_{z}^{2} x^{2}}$,

where $\xi$ is an (almost) arbitrary factor scaling the sharpness of the density gradient. The pressure tensor is given by $p_{x x}=p_{z z}=n T$ and

$$
\begin{aligned}
p_{y y}= & n T\left[1+\frac{-2 m T N_{0} \xi}{q^{2}} e^{-\xi B_{z}^{2} x^{2}} \times\right. \\
& \left.\left\{\frac{1-2 \xi B_{z}^{2} x^{2}}{n_{0}+N_{0} e^{-\xi B_{z}^{2} x^{2}}}+\frac{2 x^{2} \xi N_{0} e^{-\xi B_{z}^{2} x^{2}}}{\left(n_{0}+N_{0} e^{-\xi B_{z}^{2} x^{2}}\right)^{2}}\right\}\right] .
\end{aligned}
$$

If $n_{0}=0, p_{y y}$ becomes very simple. Let us define $h$ by $\xi=q^{2} / 2 m T_{i} h^{2}$; it caracterizes the size of the structure compared to the ion Larmor radius because (see Eq. (32)) $n=N_{0} \exp \left(x / h \rho_{i}\right)^{2}$. The $p_{y y}$ pressure component is

$p_{y y}=n T\left[1-\frac{2 m T \xi}{q^{2}}\right]=n T\left[1-\frac{1}{h^{2}}\right]$.

As long as the structure is large compared to the ion Larmor radius $(h \gg 1)$, the pressure is nearly scalar. If the structure is of the order of a few ion gyroradii $(h \sim 1)$, the pressure tensor becomes strongly non gyrotropic, and $p_{y y}<p_{x x}=p_{z z}$. (The fact that $p_{y y}$ becomes negative for $h<1$ confirms that the structure cannot be smaller than one ion gyroradius.)

\section{Conclusion}

The above computation of the pressure tensor associated with tangential equilibria shows that we must be very carefull when using a set of fluid equations to describe a tangential plasma structure: even in the simple cases given here for illustration, the pressure tensor is non isotropic and non gyrotropic. Moreover, when the magnetic field direction changes (a very common situation with tangential discontinuities), the off-diagonal terms cannot be neglected: this reinforces the non gyrotropic character of the plasma pressure tensor. Therefore, considering only the $p_{\|}$and $p_{\perp}$ components of a diagonal pressure tensor cannot provide a good description of the plasma. This is not a problem for a static description, because only the $p_{x x}$ component plays a role in the fluid equations of the equilibrium. But when the stability of the equilibrium or the magnetic reconnection are investigated, for instance, through a perturbative analysis, the other components of the equilibrium pressure tensor come into action. Not considering them can be misleading.

Acknowledgements. The author gratefully acknowledges stimulating and useful discussions with G. Belmont, V. Génot, and A. Roux.

Topical Editor T. Pulkkinen thanks two referees for their help in evaluating this paper. 


\section{References}

Attico, N. and Pegoraro, F: Periodic equilibria of the vlasovMaxwell system, Physics of Plasmas, 6, 767-770, 1999.

Channell, P. J.: Exact Vlasov-Maxwell equilibria with sheared magnetic fields, Physics of Fluids, 19, 1541-1545, 1976.

Dunlop, M. W., Balogh, A., and Glassmeier, K.-H.: Four-point Cluster application of magnetic field, analysis tools: The discontinuity analyzer, J. Geophys. Res. S. P., 24-1, 2002.

Harris, E. G.: On a plasma sheath separating regions of oppositely directed magnetic field, Il Nuovo Cimento, 23, 115-121, 1962.

Hilgers, A., Holback, B., Holmgren, G., and Bostrom, R.: Probe measurements of low plasma densities with applications to the auroral acceleration region and auroral kilometric radiation sources, J. Geophys. Res. S. P., 97, 8631-8641, 1992.
Mottez, F.: Exact nonlinear analytic Vlasov-Maxwell tangential equilibria with arbitrary density and temperature profiles, Phys. of Plasmas, 10, 2501-2508, 2003.

Petrukovich, A. A., Baumjohann, W., Nakamura, R., Balogh, A., Mukai, T., Glassmeier, K.-H., Rème, H., and Klecker, B.: Plasma sheet structure during strongly northward IMF, J. Geophys. Res. S. P., 22-1, 2003.

Roth, M., de Keyser, J., and Kuznetsova, M. M.: Vlasov theory of the equilibrium structure of tangential discontinuities in space plasmas, Space Science Reviews, 76, 251-317, 1996. 